\title{
Monitoreo de pastos marinos en Perezoso, Cahuita, Costa Rica (sitio CARICOMP)
}

\author{
Ana C. Fonseca E. ${ }^{1}$, Vanessa Nielsen M. ${ }^{2}$ \& Jorge Cortés ${ }^{1,2}$ \\ 1 Centro de Investigación en Ciencias del Mar y Limnología (CIMAR), Universidad de Costa Rica (UCR), 2060, San \\ José, Costa Rica;. Fax (506) 207-3280; afonseca@cariari.ucr.ac.cr \\ 2 Escuela de Biología, Universidad de Costa Rica (UCR), 2060, San José, Costa Rica; vnielsen@cariari.ucr.ac.cr
}

Recibido 31-X-2005. Corregido 16-V-2006. Aceptado 08-XII-2006.

\begin{abstract}
Seagrass monitoring at Perezoso, Cahuita, Costa Rica (CARICOMP site). The seagrass of Perezoso (Cahuita National Park, Caribbean coast of Costa Rica) was monitored using the CARICOMP protocol. Productivity $\left(2.7 \pm 1.15 \mathrm{~g} / \mathrm{m}^{2} / \mathrm{d} ; \mathrm{n}=74\right)$ was intermediate, compared to other Caribbean sites. Total biomass was intermediate to high (750-1500 g/ $\mathrm{m}^{2}$ ) at most CARICOMP sites (Colombia, Cuba, Mexico, Puerto Rico and Venezuela) including Costa Rica $\left(822.8 \pm 391.84 \mathrm{~g} / \mathrm{m}^{2} ; \mathrm{n}=32\right)$. Turnover rates were high $(5.5 \pm 1.36 \%$; $=74)$ compared to what was found in March and August at other sites. Shoot densities average 725 shoots $/ \mathrm{m}^{2}$, in the Caribbean region, while in Costa Rica the value was higher $\left(1184 \pm 335.5\right.$ shoots $\left./ \mathrm{m}^{2}\right)$. Average leaf length and width in the entire region were $14.4 \mathrm{~cm}$ and $10.6 \mathrm{~mm}$, respectively, similar to what we found, but leaf area index average $3.4 \mathrm{~m}^{2} \mathrm{~m}^{-2}$, higher than what was found in Costa Rica $\left(0.92 \mathrm{~m}^{2} \mathrm{~m}^{-2}\right)$. At Cahuita, seagrass productivity was significantly lower in March 2005 compared with the previous six years, and biomass has decreased with time. Seagrass productivity and biomass are being affected by the maximum temperatures, which increased by almost $10^{\circ} \mathrm{C}$ from 1999 to 2005 , and show a high negative correlation. Turnover rate and temperature were not correlated. Recreational boating, swimming and nutrient loading from deforested lands in the coast, the upstream rivers and local pollution are potential sources of impact to the seagrass beds at Cahuita. Rev. Biol. Trop. 55 (1): 55-66. Epub 2007 March. 31.
\end{abstract}

Key words: seagrass, monitoring, CARICOMP, Thalassia testudinum, Parque Nacional Cahuita, Caribbean, Costa Rica, biomass, productivity, turnover rate.

Los pastos marinos son productores primarios en aguas someras tropicales, y constituyen uno de los ecosistemas más productivos de los océanos del mundo. Estas praderas marinas pueden estar asociadas a otros ecosistemas costeros como los arrecifes de coral y los manglares (Ogden y Gladfelter 1983). Los pastos marinos se han deteriorado por perturbaciones naturales y humanas, especialmente la eutroficación de las aguas costeras (Short y Burdick 1996, Short y Wyllie-Echeverria 1996).

A nivel mundial se han reportado 60 especies de pastos marinos, en el mar Caribe y el Golfo de México se han documentado nueve especies. Particularmente, en la costa Caribe de Costa Rica se han reportado cuatro especies, Thalassia testudinum (Banks ex König 1805), Syringodium filiforme (Kützing in Hohenacker 1852-1962), Halophila decipiens (Ostenfeld 1902) y Halodule wrightii (Ascherson 1868) y dos especies en nuestra costa del Pacífico Halophila bailionis (Asch 1874) y Ruppia maritima (Linnaeus 1737) (Dawson 1962, Wellington 1972, 1973, 1974, Cortés 2001).

Con la intención de determinar el estado de los pastos marinos se estableció una estación permanente de monitoreo en el Parque Nacional Cahuita, costa Caribe de Costa Rica, de acuerdo al protocolo de "Productividad Marino Costera del Caribe (CARICOMP)". En Cahuita, $40 \%$ de los pastos marinos 
murieron durante el terremoto de 1991, sin embargo, dos años después algunas poblaciones de pastos recuperaron los niveles originales de cobertura (Cortés et al. 1994).

Existen diferentes publicaciones de pastos marinos para el mar Caribe (Gessner 1971, Patriquin 1973, Buesa 1974, 1975, Greenway 1974, Gallegos et al. 1993, Van Tussenbroek, 1995, 2002), pero muy pocas para Costa Rica. Wellington (1972) analizó al género Thalassia en su descripción ecológica del ambiente marino del Parque Nacional Cahuita (PNC). La densidad, la biomasa y la productividad de los pastos de Cahuita fueron medidos por primera vez en 1995, usando el protocolo CARICOMP (Cortés 1998), pero fue en 1999 cuando se estableció la estación permanente de Perezoso con el propósito de evaluar cambios espaciales y temporales a largo plazo. Algunos datos preliminares de biomasa, productividad y densidad de T. testudinum de otros sitios dentro del PNC, medidos entre marzo y abril del 2000, ya han sido publicados (Paynter et al. 2001). Además, desde agosto de 2003, comenzó la recopilación de información adicional sobre fenología y otros aspectos ecológicos de T. testudinum (Nielsen y Cortés, no publ.).

El objetivo de este estudio es analizar los resultados del monitoreo de biomasa, productividad y tasa de recambio de $T$. testudinum en la estación de Perezoso, Cahuita, de 1999 al 2005.

\section{MATERIALES Y MÉTODOS}

Descripción del área de estudio: el Parque Nacional Cahuita, en la costa Caribe de Costa Rica, provincia de Limón, es un área protegida creada en 1970 con una extensión de 22400 ha; de las cuales 600 están cubiertas por el arrecife de franja más extenso y estudiado de Costa Rica (Cortés 1998, Fonseca 2003, Cortés y Jiménez 2003). El clima presenta dos estaciones lluviosas, de noviembre a marzo y de junio a agosto (Herrera 1984). Las mareas son mixtas, pero principalmente diurnas, con un intervalo pequeño de 30 a $50 \mathrm{~cm}$. Las olas vienen del noreste entre enero y junio y del este de julio a diciembre, dependiendo de la posición de la Zona de Convergencia InterTropical. Las corrientes fluyen de noroeste a sureste con pequeños remolinos en dirección ciclónica. Estas corrientes transportan sedimentos terrestres derivados de tierras ribereñas y costeras deforestadas (Cortés y Risk 1985, Cortés 1994).

Los lechos de pastos marinos se ubican a lo largo de la laguna del arrecife a profundidades entre 0.5 y $3 \mathrm{~m}$ (Risk et al. 1980). Algunos parches son mono-específicos de $T$. testudinum o $S$. filiformes y en la mayoría de los casos, están formados por ambas especies (Cortés y Guzmán 1985).

El protocolo de pastos marinos se estableció en Costa Rica en 1999. La estación permanente es conocida por los pescadores locales como "Perezoso" (9॰44'13.3”N, 82॰48'24”W) (Fig. 1), $500 \mathrm{~m}$ al norte de la quebrada con ese nombre. Este lecho de pastos está a menos de 1 $\mathrm{m}$ de profundidad y está dominado por T. testudinum, mezclado con $S$. filiforme.

Metodología: con la intención de comparar los resultados del monitoreo en Costa Rica con los de otros países del Caribe, se utilizó la metodología estándar de CARICOMP para medir la biomasa, la productividad, la tasa de recambio y la densidad de los pastos marinos (http://isis.uwimona.edu.jm/centres/ $\mathrm{cms} /$ caricomp/carinew.htm).

La comunidad local fue educada acerca de la importancia de los pastos marinos y la investigación científica, y entrenada para ayudar en las colectas de información, especialmente el marcaje de las plantas para medidas de crecimiento. El primer año, de marzo de 1999 a marzo de 2000, se tomaron muestras de crecimiento de pastos una vez al mes para determinar la productividad estacional. Sin embargo, los datos de diciembre, enero, febrero y mayo no se pudieron cuantificar por la alta turbidez del agua. Comparando los resultados de los meses en que fueron colectados los datos, julio mostró la productividad más alta y marzo la menor. Debido a que las condiciones del agua en julio son muy malas, la biomasa y 


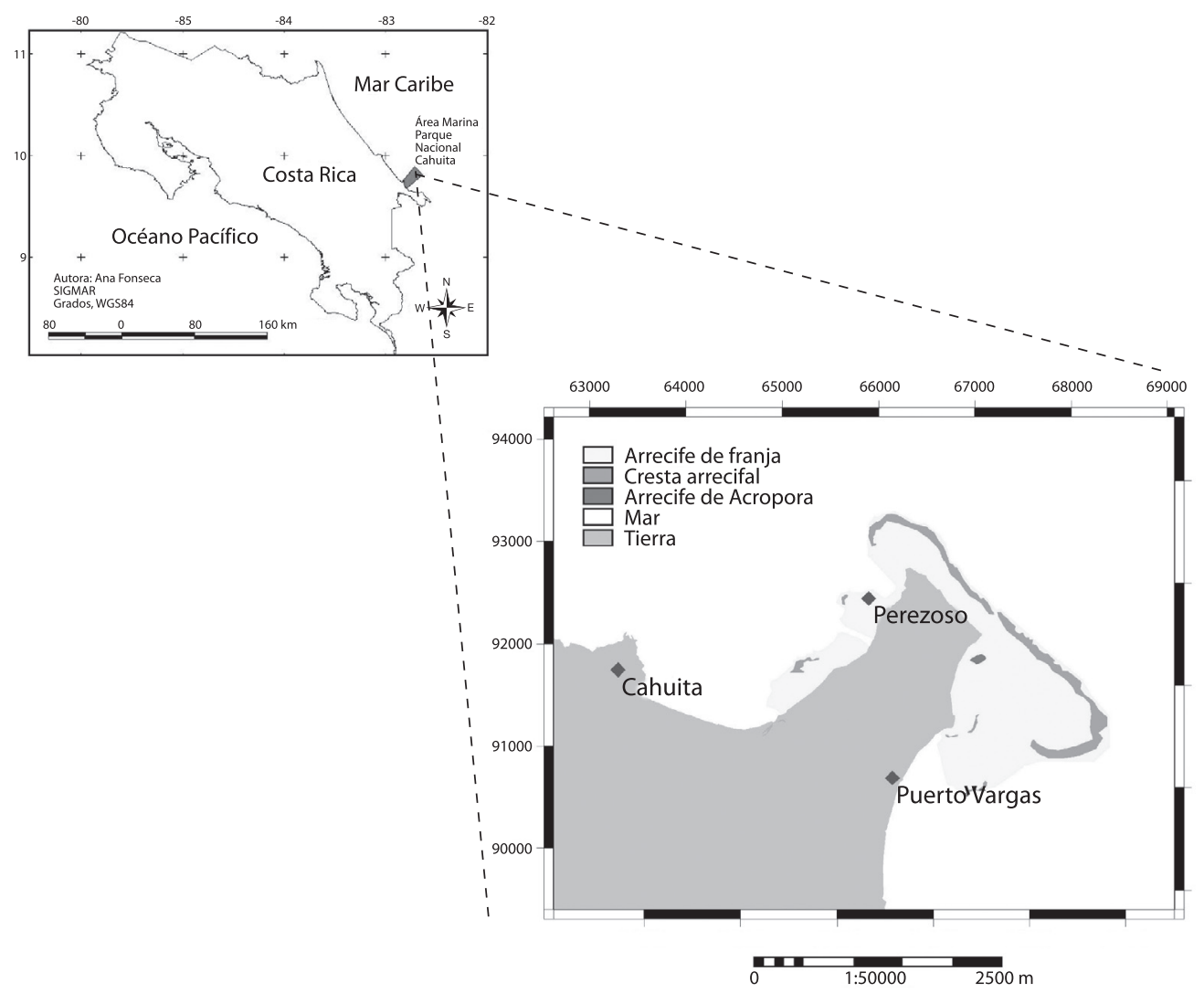

Fig. 1. Mapa de localización de Perezoso, sitio de monitoreo de pastos marinos, CARICOMP.

la productividad fueron evaluadas una vez al año en el mes de marzo, desde 1999 hasta el 2005. La temperatura del agua se midió con un sensor submarino tipo Stow Away a intervalos de $15 \mathrm{~min}$.

Todos los juegos de datos de biomasa, productividad, densidad y tasa de recambio fueron analizados para ver si seguían una distribución normal y si había heterogeneidad en las varianzas. Fue necesario transformar, los datos de productividad y tasa de recambio por medio del logaritmo en base 10. Los datos no se ajustaron al supuesto de simetría compuesta por lo que no se pudo aplicar un Análisis de Mediciones Repetidas. Para todos los datos se utilizó el análisis de varianzas (ANOVA, $\mathrm{p}<0.05$ ), con pruebas posteriores de Bonferroni o Tukey dependiendo del tamaño de las muestras (Underwood 1997).

\section{RESULTADOS}

Los lechos someros de pastos marinos en Perezoso, están dominados por T. testudinum mezclada con $S$. filiforme. Varias especies de coral, especialmente Manicina areolata (Linnaeus 1758) y Porites porites (Pallas 1766), y las algas Dictyota, Amphiroa, Laurencia y Halimeda coexisten entre estas plantas. La longitud promedio de las hojas de T. testudinum en 2000 fue de $17.54 \pm 7.47 \mathrm{~cm}$, el ancho promedio fue de $9.86 \pm 1.99 \mathrm{~mm}$, el área promedio fue de $9.68 \pm 10.76 \mathrm{~cm}^{2}$, y el índice de área foliar fue de $0.92 \pm 0.64 \mathrm{~m}^{2}$ hoja $/ \mathrm{m}^{2}$ superficie. Se observó que la floración ocurrió entre marzo y junio.

Productividad estacional: entre 1999 y 2000, julio mostró un pico significativo de productividad ( $\mathrm{F}=6.38, \mathrm{gl}=8.95, \mathrm{p}<0.05$; Fig. 2$)$ y tasa de recambio $(\mathrm{F}=32.18, \mathrm{gl}=8.95, \mathrm{p}<0.05$; 


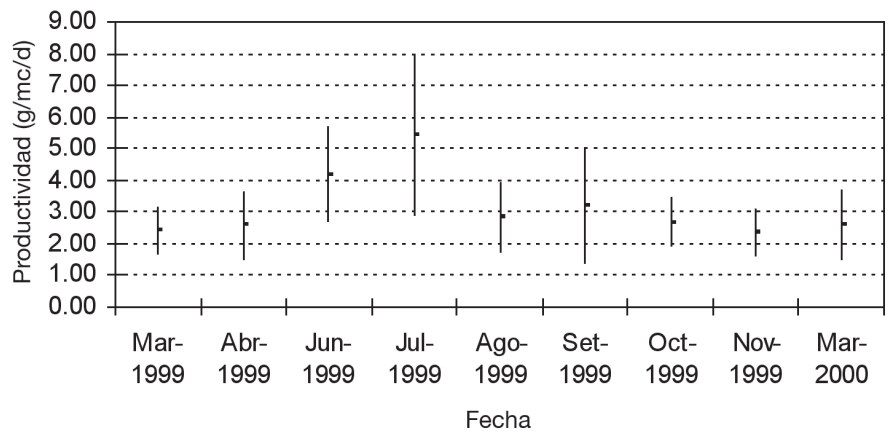

Fig. 2. Productividad de Thalassia testudinum en Perezoso. Cahuita, Costa Rica. Fig. 2. Thalassia testudinum productivity at Perezoso. Cahuita, Costa Rica.

Fig. 3), siendo la productividad de marzo significativamente más baja que en junio y julio (Fig. 2). El mes de marzo fue seleccionado para las evaluaciones anuales. En términos de densidad, abril de 1999 fue el mes con mayor densidad en comparación con setiembre 1999 y marzo $2000(\mathrm{~F}=3.65, \mathrm{gl}=8.95$, $\mathrm{p}<0.05$; Fig. 4) (Prueba Bonferroni en Apéndices 1 a 3).

La productividad, tasa de recambio y densidad anual promedio de $T$. testudinum en la estación permanente Perezoso, muestreada en marzo y julio de 1999 a 2005, se presenta en el Cuadro 1. No se apreciaron tendencias evidentes en el tiempo. La productividad resultó significativamente más baja en marzo 2005 $(\mathrm{F}=3.77, \mathrm{gl}=4.47, \mathrm{p}<0.05)$. La tasa de recambio fue significativamente más alta en marzo de $2000(\mathrm{~F}=7.74, \mathrm{gl}=4.47, \mathrm{p}<0.05)$. La densidad de pastos fue significativamente más baja en marzo de 2000 en comparación con marzo de 1999 y marzo de $2001 \quad(\mathrm{~F}=4.25, \mathrm{gl}=4.47$, $\mathrm{p}<0.05$ ) (Pruebas Bonferroni en Apéndices 4 a 6).

Biomasa: la biomasa promedio de pastos (T. testudinum) en Perezoso, en marzo de 1999 a 2005 se muestra en el Cuadro 2. La biomasa disminuyó a lo largo del tiempo $(\mathrm{F}=3.84, \mathrm{gl}=4.23$, $\mathrm{p}<0.05)$, y resultó significativamente menor en 2005 que en 1999, sin diferencias significativas entre los otros muestreos (Prueba Tukey en Apéndice 7).

Temperatura del agua en lechos de pastos marinos: la temperatura del agua varió entre 25.3 a $39.6{ }^{\circ} \mathrm{C}$. El valor máximo de temperatura

\section{CUADRO 1}

Productividad promedio anual ( \pm desv. est.), tasa de recambio y densidad de Thalassia testudinum Perezoso, Cahuita, 1999 a 2005

TABLE 1

Mean yearly productivity ( \pm standard deviation), turn-over rate and density of Thalassia testudinum Perezoso, Cahuita, 1999 to 2005

\begin{tabular}{lccc}
\multicolumn{1}{c}{ Año } & $\begin{array}{c}\text { Productividad } \\
\left(\mathrm{g} / \mathrm{m}^{2} / \text { día }\right)\end{array}$ & $\begin{array}{c}\text { Tasa de recambio por biomasa } \\
\text { de planta por día }(\%)\end{array}$ & $\begin{array}{c}\text { Densidad } \\
\left(\# \text { plantas } / 200 \mathrm{~cm}^{2}\right)\end{array}$ \\
Marzo $1999(\mathrm{n}=12)$ & $2.42 \pm 0.74$ & $4.15 \pm 0.84$ & $29.33 \pm 6.27$ \\
Julio $1999(\mathrm{n}=12)$ & $5.42 \pm 2.53$ & $8.66 \pm 1.20$ & $23.75 \pm 8.28$ \\
Marzo 2000 $(\mathrm{n}=12)$ & $2.60 \pm 1.09$ & $7.03 \pm 2.26$ & $20.75 \pm 8.38$ \\
Julio 2000 $(\mathrm{n}=10)$ & $2.02 \pm 0.61$ & $7.45 \pm 1.20$ & $19.20 \pm 3.76$ \\
Marzo 2001 $(\mathrm{n}=12)$ & $2.78 \pm 1.86$ & $3.98 \pm 2.50$ & $29.33 \pm 6.58$ \\
Marzo 2002 & - & - & - \\
Marzo 2003 $(\mathrm{n}=12)$ & $2.32 \pm 0.92$ & $4.09 \pm 1.37$ & $22.17 \pm 6.09$ \\
Marzo 2004 & - & - & - \\
Marzo 2005 $(\mathrm{n}=4)$ & $1.03 \pm 0.34$ & $3.31 \pm 0.13$ & $21.25 \pm 7.63$
\end{tabular}




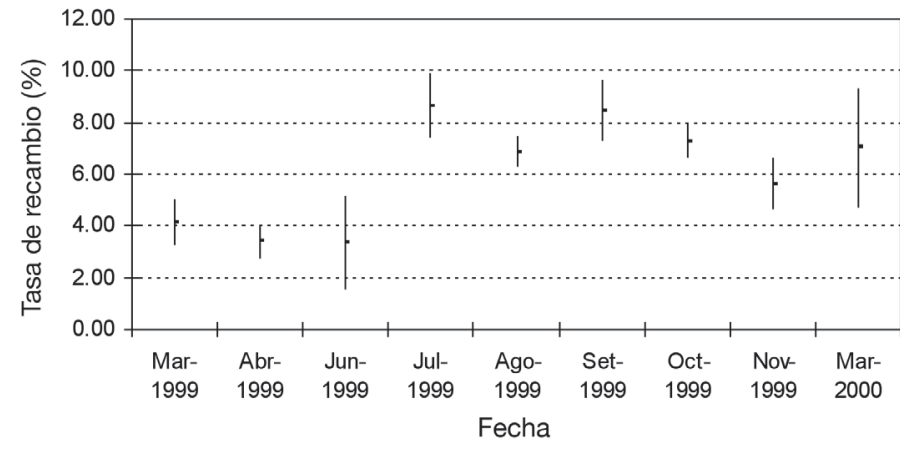

Fig. 3. Tasa de recambio de Thalassia testudinum en Perezoso. Cahuita, Costa Rica. Fig. 3. Thalassia testudinum turn-over rate at Perezoso. Cahuita, Costa Rica.

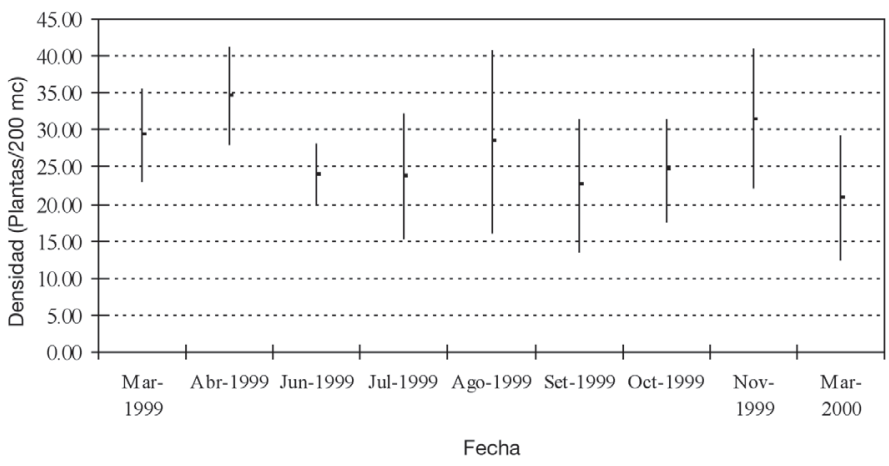

Fig. 4. Densidad de Thalassia testudinum en Perezoso. Cahuita, Costa Rica. Fig. 4. Thalassia testudinum density at Perezoso. Cahuita, Costa Rica. se registró en 2003. La temperatura mínima del agua fue medida en 2001 (Cuadro 3).

La biomasa de pastos marinos mostró una alta correlación con la temperatura promedio, mínima y especialmente con la máxima. La productividad también resultó altamente correlacionada con la temperatura máxima (Cuadro 4).

\section{DISCUSIÓN}

Las diferencias estacionales en la densidad, biomasa y productividad primaria de $T$. testudinum, con valores máximos en verano y mínimos en invierno, ha sido registrada por Ziemann (1974a,b, 1975), Thorhaug y Roesler (1977) y Van Tussenbroek (1995) en Estados Unidos y México. Kaldy y Dunton (2000) encontraron que las fluctuaciones estacionales son los controles dominantes
CUADRO 2

Biomasa promedio ( \pm desv. est, $n=4$ ) de Thalassia testudinum. Perezoso, Cahuita. marzo 1999 a 2005

TABLE 2

Mean biomass ( \pm standard deviation, $n=4$ ) of Thalassia testudinum. Perezoso, Cahuita, 1999 to 2005

\section{Año \\ Biomasa $\left(\mathrm{g} / \mathrm{m}^{2}\right)$}

Marzo $1999(\mathrm{n}=8)$

$1128.8 \pm 254.4$

Marzo $2000(\mathrm{n}=4)$

$1191.1 \pm 193.1$

Marzo $2001(\mathrm{n}=4)$

$1140.7 \pm 292.4$

Marzo $2003(\mathrm{n}=8)$

$832.1 \pm 229.7$

Marzo $2004(\mathrm{n}=4)$

$738.1 \pm 177.1$

Marzo $2005(n=4)$
$651.3 \pm 349.2$

\section{CUADRO 3}

Temperatura del agua promedio ( \pm desv. est.) anual en los pastos marinos Perezoso, Cahuita, 2000 a 2004

TABLE 3

Meanyearly water temperature ( \pm standard deviation) in Perezoso, Cahuita, 1999 to 2005.

$\begin{array}{cccc}\text { Año } & \begin{array}{c}\text { Temperatura } \\ \text { promedio } \\ \left({ }^{\circ} \mathrm{C}\right)\end{array} & \begin{array}{c}\text { Temperatura } \\ \text { mínima } \\ \left({ }^{\circ} \mathrm{C}\right)\end{array} & \begin{array}{c}\text { Temperatura } \\ \text { máxima } \\ \left({ }^{\circ} \mathrm{C}\right)\end{array} \\ 1999 & 26.1 \pm 0.72 & 22.9 \pm 0.49 & 29.4 \pm 0.96 \\ 2000 & 29.2 \pm 1.72 & 25.9 \pm 1.35 & 35.7 \pm 1.40 \\ 2001 & 27.8 \pm 1.34 & 25.3 \pm 1.27 & 32.0 \pm 1.07 \\ 2002 & - & - & - \\ 2003 & 30.0 \pm 1.83 & 26.8 \pm 0.31 & 39.6 \pm 2.55 \\ 2004 & 29.3 \pm 0.03 & 25.4 \pm 0.80 & 39.3 \pm 2.19\end{array}$


CUADRO 4

Correlaciones entre variables de pastos marinos

y temperatura $(n=4)$

TABLE 4

Temperature and seagrass variables correlations $(n=4)$

$\begin{array}{lccc} & \begin{array}{c}\text { Temperatura } \\ \text { promedio } \\ \left({ }^{\circ} \mathrm{C}\right)\end{array} & \begin{array}{c}\text { Temperatura } \\ \text { mínima } \\ \left({ }^{\circ} \mathrm{C}\right)\end{array} & \begin{array}{c}\text { Temperatura } \\ \text { máxima } \\ \left({ }^{\circ} \mathrm{C}\right)\end{array} \\ \begin{array}{l}\text { Productividad } \\ \left(\mathrm{g} / \mathrm{m}^{2} / \text { día }\right)\end{array} & -0.44 & -0.13 & -0.74 \\ \begin{array}{l}\text { Tasa de } \\ \text { recambio }(\%)\end{array} & 0.29 & 0.36 & 0.03 \\ \begin{array}{l}\text { Biomasa } \\ \left(\mathrm{g} / \mathrm{m}^{2}\right)\end{array} & -0.87 & -0.66 & -0.97\end{array}$

del crecimiento y producción de $T$. testudinum en lagunas costeras subtropicales (Baja Laguna Madre, Texas). Las condiciones de luz y temperatura más estables típicas de las regiones tropicales se reflejan en una biomasa de pastos relativamente uniforme a lo largo del año (Duarte 1989, Hillman et al. 1989). Sin embargo, se han observado fluctuaciones estacionales considerables en los trópicos. Los cambios moderados en el período diurno de luz y temperatura, explican parcialmente dichas observaciones (Mellors et al. 1993; Lanyon y Marsh 1995). En el presente estudio, la productividad de los pastos resultó significativamente menor en marzo, a mediados del invierno, y mayor en julio a finales del invierno.

La biomasa y la productividad de los pastos disminuyeron con el tiempo, afectadas aparentemente por las altas temperaturas, las cuales aumentaron casi $10{ }^{\circ} \mathrm{C}$ de 1999 a 2005 . Ambas variables muestran una fuerte correlación inversa con la temperatura (-0.7). La tasa de recambio y temperatura no resultaron correlacionadas. El crecimiento foliar, la biomasa y productividad primaria también mostraron una correlación inversa significativa con la temperatura del agua en otro arrecife coralino lagunar en el Caribe (Van Tussenbroek 1995). Las plantas corren el riesgo de experimentar estrés termal que puede ser dañino y causar la muerte (Ziemann y Wood 1975). El mayor efecto de la temperatura en los pastos es fisiológica, relacionado con la tolerancia termal individual de cada especie y las temperaturas óptimas para fotosíntesis, respiración y crecimiento (Short et al. 2001).

Entre marzo y abril de 1995 (Cortés 1998), en otro parche de pastos en la laguna frente a punta Cahuita, se encontró usando la misma metodología, que la densidad de Thalassia era $1035 \pm 38$ plantas $/ \mathrm{m}^{2}$ y su productividad era $3.0 \pm 3.20 \mathrm{~g} / \mathrm{m}^{2} / \mathrm{d}$. En 1999, (Marzo-Abril), se registró una densidad de 1418 plantas $/ \mathrm{m}^{2}$ y una productividad de $2.4 \mathrm{~g} / \mathrm{m}^{2} / \mathrm{d}$ en Perezoso (Paynter et al. 2001). Estos datos son similares a lo que se encontró en el presente estudio (1 $184 \pm 335.5$ plantas $/ \mathrm{m}^{2}$ y $2.7 \pm 1.15 \mathrm{~g} / \mathrm{m}^{2} / \mathrm{d}$ respectivamente).

La biomasa total resultó mayor en Belize (>4 $000 \mathrm{~g}$ peso $\mathrm{seco} / \mathrm{m}^{2}$ ), menor en Tobago y Curasao $\left(200-500 \mathrm{~g} / \mathrm{m}^{2}\right)$, y de intermedio a alto $\left(750-1500 \mathrm{~g} / \mathrm{m}^{2}\right)$ en todos los otros sitios CARICOMP (Colombia, Cuba, México, Puerto Rico y Venezuela) incluyendo Costa Rica $\left(822.8 \pm 391.84 \mathrm{~g} / \mathrm{m}^{2} / \mathrm{a}, \mathrm{n}=32\right)$. La productividad en toda la región Caribe varía de menos de $0.5 \mathrm{~g} / \mathrm{m}^{2} / \mathrm{d}$ (Bahamas) a más de $5 \mathrm{~g} / \mathrm{m}^{2} / \mathrm{d}$ (Venezuela) y las tasas de recambio tienen un promedio de $3.9 \% \mathrm{~d}^{-1}$. En Costa Rica, la productividad $\left(2.7 \pm 1.15 \mathrm{~g} / \mathrm{m}^{2} / \mathrm{d}, \mathrm{n}=74\right)$ es intermedia, comparada con otros sitios, y similar a lo que se encontró en Colombia. La tasa de recambio es alta $(5.5 \pm 1.36 \%, \mathrm{n}=74)$ comparada con lo que se encontró en marzo en otros sitios; valores similares se encontraron entre marzo y junio en Barbados, México, Bermudas y Puerto Rico. Aparentemente, la tasa de recambio es alta en lechos de pastos que crecen a lo largo de las costas continentales o islas grandes sujetas a gran descarga de nutrientes terrestres, lo cuál los hace más vigorosos. La densidad promedio en la región Caribe fue de 725 plantas $/ \mathrm{m}^{2}$, por lo que en Costa Rica está por encima del promedio (1 $184 \pm 335.5$ plantas $/ \mathrm{m}^{2}$ ). El largo $\mathrm{y}$ ancho de hoja promedio en toda la región fue de $14.4 \mathrm{~cm}$ y $10.6 \mathrm{~mm}$, respectivamente, similar a lo que encontramos en este estudio, pero el índice de área foliar tuvo un promedio 
de $3.4 \mathrm{~m}^{2} \mathrm{~m}^{-2}$, más alto de lo que se encontró en Costa Rica $\left(0.92 \mathrm{~m}^{2} \mathrm{~m}^{-2}\right)$ (CARICOMP 1997, 2000, Zieman et al. 1997).

Debido a que los lechos de pastos de Perezoso se encuentran en la laguna del arrecife de franja de Cahuita, ambos ecosistemas interactúan estrechamente, y los pastos sirven como ambiente de reproducción y alimentación para organismos arrecifales. La actividad de botes, nadadores y la descarga de nutrientes (Roder 2005) son fuentes potenciales de impacto sobre la cobertura y productividad de los pastos marinos de Cahuita, y por lo tanto sobre la actividad pesquera de las comunidades locales. Estas relaciones serán evaluadas en un futuro cercano. Se recomienda establecer los requisitos de hábitat de cada especie de pasto marino y evaluar las respuestas particulares a diferentes tipos de sedimento y calidad del agua.

\section{AGRADECIMIENTOS}

Los estudios de pastos marinos han sido posibles gracias al apoyo de CARICOMP, el Consejo Nacional de Investigaciones Científicas y Tecnológicas (CONICIT), United Nations Environmental Program-Jamiaca (UNEP), USISRAEL Cooperative Development Research Program y la Vicerrectoría de Investigación, Universidad de Costa Rica. Un agradecimiento especial para José Saballo y toda la gente del Parque Nacional Cahuita, Cindy Fernández, Justo López, la familia Mairena, y muchos asistentes y administradores del CIMAR por su apoyo.

\section{RESUMEN}

El pasto marino de Perezoso en el Parque Nacional Cahuita, costa Caribe de Costa Rica, se está monitoreando con el protocolo de CARICOMP. La productividad $\left(2.7 \pm 1.15 \mathrm{~g} / \mathrm{m}^{2} / \mathrm{d}, \mathrm{n}=74\right)$ resultó intermedia, comparada con otros sitios del Caribe. La biomasa total resultó de intermedia a alta $\left(750-1500 \mathrm{~g} / \mathrm{m}^{2}\right)$ en la mayoría de los sitios CARICOMP (Colombia, Cuba, Mexico, Puerto Rico y Venezuela) incluyendo Costa Rica $\left(822.8 \pm 391.84 \mathrm{~g} / \mathrm{m}^{2}\right.$, $\mathrm{n}=32)$. Las tasas de recambio son altas $(5.5 \pm 1.36 \%, \mathrm{n}=74)$ comparadas con lo que se encontró en marzo y agosto en otros sitios. La densidad promedio es de 725 plántulas $/ \mathrm{m}^{2}$, en la región Caribe, mientras que en Costa Rica resultó mayor (1 $184 \pm 335.5$ plántulas $/ \mathrm{m}^{2}$ ). El largo y ancho de hoja promedio en toda la región es de $14.4 \mathrm{~cm}$ y 10.6 $\mathrm{mm}$, respectivamente, similar a lo que se encontró en este estudio, pero el índice de área foliar promedio es de 3.4 $\mathrm{m}^{2} \mathrm{~m}^{-2}$, mayor a lo que se encontró en Costa Rica (0.92 $\mathrm{m}^{2} \mathrm{~m}^{-2}$ ). En Cahuita la productividad del pasto es significativamente menor comparado con los seis años previos, y la biomasa disminuyó en el tiempo. La productividad y biomasa de los pastos se está viendo afectada por las temperaturas máximas que aumentaron casi $10{ }^{\circ} \mathrm{C}$ de 1999 a 2005, y muestran una alta correlación negativa. La tasa de recambio y la temperatura no mostraron correlación. La actividad por botes, nadadores y la alta carga de nutrientes proveniente de la contaminación local por aguas negras, la deforestación y las actividades agrícolas en tierras costeras y rivereñas son fuentes potenciales de impacto en los pastos marinos de Cahuita.

Palabras clave: pastos marinos, monitoreo, CARICOMP, Thalassia testudinum, Parque Nacional Cahuita, Caribe, Costa Rica, biomasa, productividad, tasa de recambio.

\section{REFERENCIAS}

Buesa, R.J. 1974. Population and biological data on turtle grass (Thalassia testudinum Konig, 1805) on the northwestern Cuban Shelf. Aquacul. 4: 207-226.

Buesa, R.J. 1975. Populations biomass and metabolic rates of marine angiosperms on the northwestern Cuban Shelf. Aquat. Bot. 1: 11-23.

Cortés, J. 1994. A reef under siltation stress: a decade of degradation, p 240-246. In R.N. Ginsburg (ed.). Proc. Colloquium on Global Aspects of Coral Reefs: Health, Hazards and History, 1993. RSMAS, Univ. Miami, Miami, Florida, EEUU.

Cortés, J. 1998. Cahuita and Laguna Gandoca, Costa Rica, p 107-113. In B. Kjerve (ed.). CARICOMP, Caribbean Coral Reef, Seagrass and Mangrove Sites. UNESCO, Paris, Francia.

Cortés, J. 2001. Requiem for an eastern Pacific seagrass bed. Rev. Biol. Trop. 49: 273-278.

Cortés, J. \& H.M. Guzmán. 1985. Arrecifes coralinos de la costa Atlántica de Costa Rica. Brenesia 23: 275-292.

Cortés, J. \& C. Jiménez. 2003. Coral reefs of the Caribbean coast of Costa Rica, p 223-239. In J. Cortés (ed.). Latin American Coral Reefs. Elsevier Science, Amsterdam, Holanda. 
Cortés, J \& M.J. Risk. 1985. A reef under siltation stress: Cahuita, Costa Rica. Bull. Mar. Sci. 36: 339-356.

Cortés, J., R. Soto \& C. Jiménez. 1994. Efectos ecológicos del terremoto de Limón. Rev. Geol. Amér. Central, vol. esp. Terremoto de Limón: 187-192.

Dawson, E.Y. 1962. Additions to the marine flora of Costa Rica and Nicaragua. Pacific Nat. 3: 375-395.

Duarte, C.M. 1989. Temporal biomass variability and production/biomass relationships of seagrass communities. Mar. Ecol. Prog. Ser: 269-76.

Fonseca, A.C. 2003. A rapid assessment at Cahuita National Park, Costa Rica, 1999 (Part I: stony corals and algae), p 249-257. In J.C. Lang (ed.).Status of Coral Reefs in the Western Atlantic: Results of initial surveys, Atlantic and Gulf Rapid Reef Assessment (AGRRA) Program. Atoll Res. Bull. 496.

Gallegos, M.E., M. Merino, N. Marbá \& C.M. Duarte. 1993. Biomass and dynamics of Thalassia testudinum in the Mexican Caribbean: elucidating rhizome growth. Mar. Ecol. Prog. Ser. 95: 185-192.

Gessner, F. 1971. The water economy of the seagrass Thalassia testudinum. Mar. Biol. 10: 258-260.

Greenway, M. 1974. The effects of cropping on the growth of Thalassia testudinum (König) in Jamaica. Aquacul. 4: 199-206.

Herrera, W. 1984. Clima de Costa Rica. Universidad Estatal a Distancia (EUNED), San José, Costa Rica.

Hillman, K., D.I. Walker, A.W.D Larkum \& A.J. McComb.1989. Productivity and nutrient limitation, p 635-685. In A.W.D. Larkum, A.J. McComb \& S.A. Shepherd (eds.). Biology of Seagrasses. Elsevier, Amsterdam, Holanda.

Kaldy, J.E. \& K.H. Dunton. 2000. Above-and belowground production, biomass and reproductive ecology of Thalassia testudinum (turtle grass) in a subtropical coastal lagoon. Mar. Ecol. Prog. Ser.193: 271-283.

Lanyon, J.M. \& H. Marsh. 1995. Temporal changes in the abundance of some tropical intertidal seagrasses in North Queensland. Aquat. Bot. 217-37.

Mellors, J.E., H. Marsh \& R.G. Coles. 1993. Intra-annual changes in seagrass standing crop, Green Island, Northern Queensland. Austr. J. Mar. Freshwater. Res. 44: 33-41.
Ogden, J.C. \& E.H. Gladfelter. 1983. Coral Reefs, Seagrass Beds and Mangroves: Their Interaction in the Coastal Zones of the Caribbean. UNESCO, Paris, Francia. 133 p.

Patriquin, D.G. 1973. Estimation of growth rate, production and age of the marine angiosperm Thalassia testudinum König. Carib. J. Sci. 13: 111-124.

Paynter, C., J. Cortés \& M. Engels. 2001. Biomass, productivity and density of the seagrass Thalassia testudinum at three sites in Cahuita National Park, Costa Rica. Rev. Biol. Trop. 49: 265-272.

Risk, M.J., M.M. Murillo \& J. Cortés. 1980. Observaciones biológicas preliminares sobre el arrecife coralino en el Parque Nacional Cahuita, Costa Rica. Rev. Biol. Trop. 28: 361-382.

Roder, C. 2005. Land-based pollution on the Caribbean coast of Costa Rica: nutritional and skeletal characteristics of the reef-building coral Siderastrea siderea as a response. M.Sc. Thesis in International Studies in Aquatic Tropical Ecology. Faculty of Biology \& Chemistry, Universitat Bremen, Bremen, Alemania. $82 \mathrm{p}$.

Short, F.T. \& D.M. Burdick. 1996. Quantifying eelgrass loss in relation to housing development and nitrogen lading in Waquoit Bay, Massachusetts. Estuaries 19: $17-27$.

Short, F.T. \& S. Wyllie-Echeverria. 1996. Natural and human-induced disturbances of seagrass. Environ. Conser. 23: 17-27.

Short, F.T., R.G. Coles \& C. Pergent-Martini. 2001. Global seagrass distribution, p. 9. In F.T. Short \& R.G. Coles (eds.). Global Seagrass Research Methods. Elsevier Science, Amsterdam, Holanda.

Thorhaug, A. \& M.A. Roesler. 1977. Seagrass community dynamics in a subtropical estuarine lagoon. Aquaculture 12: 253-277.

Underwood, A. 1997. Experiments in Ecology: Their Logical Design and Interpretation Using Analysis of Variance. University of Cambridge, Cambridge, Inglaterra. $504 \mathrm{p}$.

Van Tussenbroek, B.I. 1995. Thalassia testudinum leaf dynamics in a Mexican Caribbean coral reef lagoon. Mar. Biol. 122: 33-40. 
Van Tussenbroek, B.I. 2002. Static life-table analysis and demography of the foliar shoots of the topical seagrass Thalassia testudinum. Bull. Mar. Sci. 71: 1247-1256.

Wellington, G.M. 1973. Additions to the Atlantic benthic flora of Costa Rica. Brenesia 2: 17-20.

Wellington, G.M. 1974. The benthic flora of Punta Cahuita: annotated list of species with additions to the Costa Rican Atlantic flora. Brenesia 3: 19-30.

Zieman, J.C. 1974a. Methods for the study of the growth and production of turtle grass, Thalassia testudinum König. Aquacul. 4: 139-143.

Zieman, J.C. 1974b. Quantitative and dynamic aspects of the ecology of turtle grass, Thalassia testudinum, p. 541-562. In L.E. Cronin (ed.). Recent Advances in Estuarine Research. Vol. 1. Academic, Nueva York, Nueva York, EEUU.
Zieman, J.C. 1975. Seasonal variation of turtle grass, Thalassia testudinum Konig, with reference to temperature and salinity effects. Aquat. Bot. 1: 107-123.

Zieman, J.C. \& E.J.F. Wood. 1975. Effects of thermal pollution of tropical-type estuaries, with emphasis on Biscayne Bay, Florida, p.75-98. In E.J.F. Wood \& R. Johannes (eds.). Tropical Marine Pollution. Elsevier, Amsterdam, Holanda.

Zieman J., P. Penchaszadeh, J.R. Ramirez, D. Perez, D. Bone, J. Herrera-Silveira, R.D. Sanchez-Arguelles, D. Zuniza, B. Martinez, K. Bonair, P. Alcolado, R. Laydoo, J.R. Garcia, J. Garzón-Ferreira, G. Diaz, S.R. Smith, R. Varela, K. Koltes \& J. Tschirky. 1997. Variation in ecological parameters of Thalassia testudinum across the CARICOMP Network. Proc. 8th Int. Coral Reef Symp., Panamá 1: 663-668.

\section{APÉNDICES}

\section{APÉNDICE 1}

Prueba Bonferroni para la productividad estacional de los pastos marinos. Agosto 1999 a marzo 2000

\section{APPENDIX 1}

Bonferroni test for seagrass seasonal productivity. August 1999 to March 2000

Ago-1999 Mar-1999 Abr-1999 Jun-1999 Jul-1999 Set-1999 Oct-1999 Nov-1999 Mar-2000

$\begin{array}{lcccccccc}\text { Ago-1999 } & 1.00 & 1.00 & 0.29 & 0.00^{*} & 1.00 & 1.00 & 1.00 & 1.00 \\ \text { Mar-1999 } & & 1.00 & 0.02 * & 0.00^{*} & 1.00 & 1.00 & 1.00 & 1.00 \\ \text { Abr-1999 } & & & 0.06 & 0.00^{*} & 1.00 & 1.00 & 1.00 & 1.00 \\ \text { Jun-1999 } & & & & 1.00 & 1.00 & 0.17 & 0.02^{*} & 0.03^{*} \\ \text { Jul-1999 } & & & & 0.02^{*} & 0.00^{*} & 0.00^{*} & 0.00^{*} \\ \text { Set-1999 } & & & & & 1.00 & 1.00 & 1.00 \\ \text { Oct-1999 } & & & & & & & 1.00 & 1.00 \\ \text { Nov-1999 } & & & & & & & & \\ \end{array}$

Mar-2000 


\section{APÉNDICE 2}

Prueba Bonferroni para la tasa de recambio estacional de los pastos marinos. Agosto 1999 a marzo 2000

\section{APPENDIX 2}

Bonferroni test for seagrass seasonal turn-over rate. August 1999 to March 2000

$$
\text { Ago-1999 Mar-1999 Abr-1999 Jun-1999 Jul-1999 Set-1999 Oct-1999 Nov-1999 Mar-2000 }
$$

Ago-1999

Mar-1999

Abr-1999

Jun-1999

Jul-1999

Set-1999

Oct-1999

Nov-1999

Mar-2000

\section{APÉNDICE 3}

Prueba Bonferroni para la densidad estacional de los pastos marinos. Agosto 1999 a marzo 2000

\section{APPENDIX 3}

Bonferroni test for seagrass seasonal density. August 1999 to March 2000

Ago-1999 Mar-1999 Abr-1999 Jun-1999 Jul-1999 Set-1999 Oct-1999 Nov-1999 Mar-2000

\begin{tabular}{|c|c|c|c|c|c|c|c|}
\hline Ago-1999 & 1.00 & 1.00 & 1.00 & 1.00 & 1.00 & 1.00 & 1.00 \\
\hline Mar-1999 & & 1.00 & 1.00 & 1.00 & 1.00 & 1.00 & 1.00 \\
\hline Abr-1999 & & & 0.07 & 0.06 & $0.02 *$ & 0.15 & 1.00 \\
\hline Jun-1999 & & & & 1.00 & 1.00 & 1.00 & 1.00 \\
\hline Jul-1999 & & & & & 1.00 & 1.00 & 1.00 \\
\hline Set-1999 & & & & & & 1.00 & 0.50 \\
\hline Oct-1999 & & & & & & & 1.00 \\
\hline Nov-1999 & & & & & & & \\
\hline
\end{tabular}

Mar-2000 
APÉNDICE 4

Prueba Bonferroni para la productividad anual de los pastos marinos, 1999 a 2005

APPENDIX 4

Bonferroni test for seagrass yearly productivity, 1999 to 2005

Mar-1999

Mar-1999

Mar-2000

Mar-2001

Mar-2003

Mar-2005

$$
\text { Mar-2000 }
$$

1.00

\section{APENDICE 5}

Prueba Bonferroni para la tasa de recambio anual de los pastos marinos, 1999 a 2005

\section{APPENDIX 5}

Bonferroni test for seagrass yearly turn-over rate, 1999 to 2005
Mar-2003

Mar-2005

1.00

1.00

$0.01 *$

1.00

$0.01 *$

1.00

$0.01 *$

0.03 *
Mar-2000

$0.00 *$

Mar-1999

Mar-2000

Mar-2001

Mar-2003

Mar-2005
APÉNDICE 6

Prueba Bonferroni para la densidad anual de los pastos marinos, 1999 a 2005

\section{APPENDIX 6}

Bonferroni test for seagrass yearly density, 1999 to 2005
Mar-1999

Mar-1999
Mar-2001

Mar-2003

0.15

1.00

0.15
1.00

0.00 *

1.00

1.00
Mar-1999

Mar-2000

Mar-2001

Mar-2003

Mar-2005
$0.04 *$

0.04*
Mar-2005

1.00

0.00 *

.00 
APÉNDICE 7

Prueba Tukey para la biomasa anual de los pastos marinos, 1999 a 2005

APPENDIX 7

Tukey test for yearly seagrass biomass, 1999 to 2005

Mar-1999 Mar-2001 Mar-2003 Mar-2004 Mar-2005

Mar-1999

1.00

0.18

0.13

0.04*

Mar-2001

0.32

0.21

0.09

Mar-2003

0.97

0.78

Mar-2004

0.99

Mar-2005 